See discussions, stats, and author profiles for this publication at: https://www.researchgate.net/publication/287222616

\title{
A Comparison of Multi-scale Local Binary Pattern Variants for Bark Image Retrieval
}

Conference Paper · October 2015

DOI: 10.1007/978-3-319-25903-1_66

\section{CITATIONS}

21

3 authors, including:

\section{Safia Boudra}

Université Batna 2

6 PUBLICATIONS 38 CITATIONS

SEE PROFILE
READS

677

Itheri Yahiaoui

Université de Reims Champagne-Ardenne

46 PUBLICATIONS 776 CITATIONS

SEE PROFILE

Some of the authors of this publication are also working on these related projects:

Project $\quad$ Plant Recognition View project

Project 2D shape retrieval View project 


\title{
A Comparison of Multi-Scale Local Binary Pattern Variants for Bark Image Retrieval
}

\author{
Safia Boudra ${ }^{1}$, Itheri Yahiaoui ${ }^{2}$, Ali Behloul $^{1}$ \\ ${ }^{1}$ LaSTIC, Université de Batna. Algérie. \\ \{safia.boudra, ali.behloul\}@gmail.com \\ ${ }^{2}$ CReSTIC, Université de Reims Champagne-Ardenne, France. \\ itheri.yahiaoui@univ-reims.fr
}

\begin{abstract}
With the growing interest in identifying plant species and the availability of digital collections, many automated methods based on bark images have been proposed. Bark identification is often formulated as a texture analysis problem. Among numerous approaches, Local Binary Pattern (LBP) based texture description has achieved good performances. Bark structure appearance is subject to resolution variations which can be due to a number of factors (environment, age, acquisition conditions, etc). Thus it remains a very challenging problem. In this paper, we implement and study the efficiency of different multi-scale LBP descriptors: Multi-resolution LBP (MResLBP), Multi-Block LBP (MBLBP), LBP-Filtering (LBPF), Multi-Scale LBP (MSLBP), and Pyramid based LBP (PLBP). These descriptors are compared on two bark datasets: AFF and Trunk12. The descriptors are evaluated under increasing levels of scale space. The performances are assessed using the Mean Average Precision and Recall $\backslash$ Precision curves. The results show that multi-scale LBP descriptors outperform the basic LBP and MResLBP. In our tests, we observe that the best results of LBPF and PLBP are obtained under low scale space levels. We also observe similar results for MSLBP and MBLBP across the six scales considered.
\end{abstract}

Keywords: Plant Identification, Multi-scale LBP, Texture description, Bark species recognition, Image retrieval.

\section{Introduction}

Plants play an essential role in sustaining life on our planet. In addition to providing a natural habitat, they are a constant source of oxygen, food, and medicine. Given the vast diversity and variety of plant species, there is understandably increasing interest in indentifying new and existing species in order to improve agriculture and plant production, while adding to the proof of knowledge available to researchers in the field of botany.

The classical approach to identifying and classifying plant species requires specialist domain knowledge meaning that only a taxonomist or botanist can distinguish between various species. 
However, ever a domain expert cannot be expected to know of all plant species, and recent years have seen increasing interest in automated plant species identification and classification.

Over the last decade, many automated plant species identification methods based on low-level features extracted from images of organs (leaf, flower, fruit, stem, bark ...) have been proposed; [1,2,3,4,5]. Mobile applications, Leafsnap and Pl@ntNet, have been developed aiming to achieve accurate real time identification. Recently, many studies based on bark (or stem) images have appeared [3, 4] [6,7,8,9,10].

The appearance and morphology of the bark are used by foresters and botanists to distinguish between plant species and to estimate plant age [11]. The Bark has the advantage of being the only part of the tree that is available throughout the year and it is present for almost all plant's lifetime. As bark is a rigid 3D object, its 2D image acquisition is straightforward.

Since bark shows texture properties, its automated identification is formally defined as a texture analysis problem, and researchers aim to develop relative methods to extract highly discriminative features for an accurate identification. Nonetheless, it remains a challenging problem in the image processing and computer vision field.

Bark appearance often depends on environmental conditions, tree age and the effects of plant diseases. Additionally, acquisition conditions like rescaling, uncontrolled illumination changes, branch shadow clutter can alter the image quality and the texture properties. Fig 1 illustrates some examples.

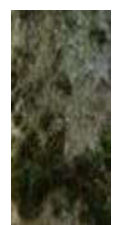

(a)

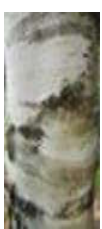

(b)

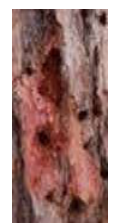

(c)

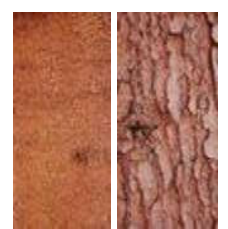

(d)

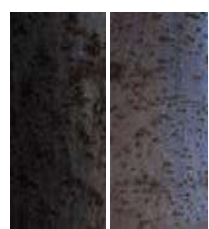

(e)

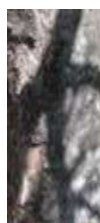

(f)

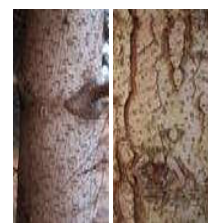

(g)

Fig. 1. Examples of the visual diversity of the surface of bark: (a) lichen, (b) peeled bark, (c) scars, (d) tree age deformation, (e) illumination change, (f) shadow clutters, (g) different texture structures at different scales.

The most significant image transformation problem in bark images is scale changes, due to the fact that image samples are taken at distinct distances from the tree trunk: texture structures taken close to the trunk are of high resolution while those far from the trunk are of low resolution. To handle this disparity, many features need to be extracted to capture texture properties at different scale levels.

Several texture descriptors [12] have been used for bark image classification and identification. Statistical features such as co-occurrence distribution were used by [7]. Huang et al [8], $[13,14]$ proposed different approaches, respectively, based on contourlet filter, Gabor filters, and Discrete Wavelet Decomposition (DW). In [4], a bag of words model was constructed with SIFT interest points. Recently, structural features such as LBP variants have been used $[9,10],[15,16]$.

Sixta et al [9], used Multi-Block Local Binary Pattern where mean filter with different sizes is applied to input images. MBLBP was the first multi-scale LBP variant 
used for bark texture analysis. Sulc et al [10] proposed a Multi-Scale LBP. Input images are Gaussian Filtered prior to the LBP operator with increasing radii. In both, histograms of all the scales considered are concatenated to form the final texture descriptor.

Motivated by the high performances achieved in the two last studies, we want to focus more closely on multi-scale LBP as efficient texture descriptor for bark texture identification.

Our work aims to quantitatively discuss and compare four different multi scale LBP approaches with filters for bark texture analysis, namely: (1) Multi-Block LBP (MBLBP) [17]with a mean filter, (2) LBP Filtering (LBPF) [18] and (3) Multi-Scale LBP (MSLBP) [10]with a low pass Gaussian filter, and (4) Pyramid-based LBP (PLPB) [19]with a pyramid transform. These methods are implemented and compared to the conventional Multi Resolution LBP (MResLBP) [20] where texture information is simply collected from single pixels at different scales rather than using filter's responses.

The remainder of this paper is structured as follows: the LBP operator and its multi-scale variants are presented in Section 2. In Section 3, a detailed description of bark datasets is given. Our bark image retrieval experiments and results are set out and discussed in Section 4. Finally, the conclusion is given in Section 5.

\section{The LBP operator and its Multi-Scale variants}

\subsection{The LBP operator}

Basic LBP [20] encodes the sign of the local difference between a central pixel $\mathrm{p}_{c}$ and its $\mathrm{P}$ neighbors $\mathrm{p}_{\mathrm{i}}$, evenly spaced on a circle of radius $\mathrm{R}$. Formally, it is given by:

$$
\mathrm{LBP}_{\mathrm{P}, \mathrm{R}}=\sum_{\mathrm{i}=0}^{\mathrm{P}-1} \mathrm{~s}\left(\mathrm{p}_{\mathrm{i}}-\mathrm{p}_{\mathrm{c}}\right) 2^{\mathrm{i}}
$$

Where $\mathrm{s}(\mathrm{x})$ is a step function; $\mathrm{s}(\mathrm{x})=1$, if $\mathrm{x}>=0$; 0 otherwise.

Once the LBP codes have been computed, a histogram is built to represent the texture image.

Another extension to the basic LBP operator considers uniform patterns; the uniformity measure was first defined by [21] as "the number of bitwise $0 / 1$ and $1 / 0$ changes when the pattern is considered circular". Based on this definition, a local binary pattern is said to be uniform if its uniformity measure takes that form $U \leq 2$.

Using this mapping, denoted by LBPu2, every uniform pattern is assigned to a single bin in the histogram and all non uniform patterns are assigned to a unique bin.

It is formally defined by:

$$
\operatorname{LBP}_{\mathrm{P}, \mathrm{R}}^{\mathrm{u} 2}=\sum_{\mathrm{i}=0}^{\mathrm{P}-1} \mathrm{~s}\left(\mathrm{p}_{\mathrm{i}}-\mathrm{p}_{\mathrm{c}}\right), \text { if } \mathrm{U}\left(\mathrm{LBP}_{\mathrm{P}, \mathrm{R}}\right) \leq 2, P+1 \text { otherwise }
$$

The uniform patterns are shown to provide fundamental local texture information properties [20]. Furthermore, it can significantly reduce the histogram length: only 59 bins with 8 samples in the local neighborhood instead of 256. 


\section{Multi Resolution LBP (MRes-LBP).}

Bark texture images are captured at various scales. Therefore, large-scale structures cannot be defined within the $3 \times 3$ space area of the original LBP.

An intuitive and efficient way to capture large-scale structures is to combine different LBP operators with increasing values of $(P, R)$, i.e. $(8,1),(16,2),(24,3)$.

Ojala's [20] multi resolution LBP (MResLBP) is based on the concatenation of three uniform LBP histograms, $\mathrm{LBP}_{8,1}^{\mathrm{u} 2}+\mathrm{LBP}_{16,2}^{\mathrm{u} 2}+\mathrm{LBP}_{24,3}^{\mathrm{u} 2}$. Fig 2 shows $\mathrm{LBP}$ operators with different configurations of $(\mathrm{P}, \mathrm{R})$.

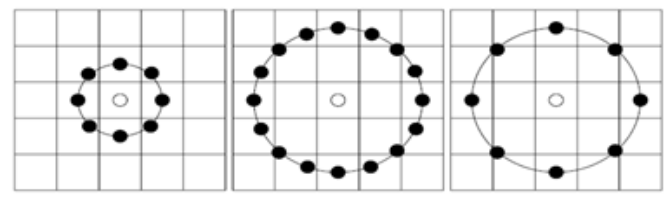

Fig. 2. LBP operator with different values of $(P, R):(8,1),(16,2)$ and $(8,2)$.

Using different LBP operators with increasing radii causes an aliasing effect and leads to noise sensitivity since the texture information is collected from a single pixel at different levels. To overcome this issue, several multi-scale LBP schemes have been proposed [10], [17,18,19] mainly based on filter responses to collect texture information from large areas at different scales. Therefore, it captures microstructures as well as macrostructures with reduced noise.

\subsection{Multi-Scale LBP}

Multi-Block Local Binary Pattern (MBLBP).

Liao [17] applied a set of mean filters at different scales to the input image. The simple point to point comparison in basic LBP was extended to a comparison between mean intensity values of square sub-blocks. The size of the filter $\mathrm{s}^{\times} \mathbf{s}$ denotes the scale of the MBLBP operators. Each filter is divided into 9 sub blocks as shown in Fig. 3. Therefore its size must be a multiple of 3 .

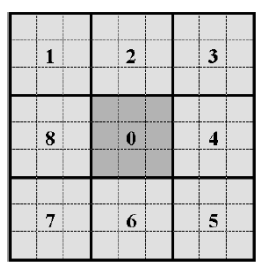

Fig. 3. MBLBP operator of size 9*9 [17]

In their work, [17] stated a new uniformity definition based on percentage distribution. The $\mathrm{n}$ patterns $(\mathrm{n}=63)$ with a high percentage distribution are labeled as uniform and the remaining patterns are labeled as non uniform. 


\section{Local Binary Pattern Filtering (LBPF).}

Following a multi-scale approach, in [18] large-scale texture patterns are detected by combining exponentially growing neighborhood radii with Gaussian low-pass filtering. Gaussian low-pass filtering is used to collect information from an area larger than a single pixel, named the "effective area". Solid circles in Fig. 4 (a) indicate a constant number of effective areas over different scales. The $\mathrm{P}$ circles $(\mathrm{P}=8)$ are tangent and have the same size.

Exponentially growing radii of the LBP operator, $R_{S}$, indicated by dotted circles in Fig. 4 (a) at level s, are given by:

$$
R_{S}=\frac{r_{S}+r_{S-1}}{2}
$$

Multi-Scale Local Binary Pattern (MSLBP).

In contrast to the scale space in [18] where LBP radii are designed so that the effective areas at different levels are tangent, in MSLBP [10] a finer scale with a step of $\sqrt{2}$ is used between LBP radii, i.e.

$$
R_{S}=R_{S-1} \sqrt{2} \text { and } R_{1}=1
$$

\section{Pyramid-Based Local Binary Patterns (PLBP).}

Qian et al [19] proposed Pyramid-based LBP. Each image at level s of the pyramid representation is obtained by convolving it with a Gaussian low pass filter, $G(x, y)$, followed by down sampling of the $\mathrm{s}-\mathrm{t}^{\text {th }}$ level image. Formally $f_{s}(x, y)$ is obtained as follows, where $f_{0}(x, y)$ is the original image:

$$
f_{s}(\mathrm{x}, \mathrm{y})=G(x, y) * f_{s-1}\left(R_{x} x+m, R_{y} y+n\right) \text {, for level } s>1
$$

Where $\mathrm{R}_{\mathrm{x}}, \mathrm{R}_{\mathrm{y}}$ are the down-sampling ratios in $\mathrm{x}$ and $\mathrm{y}$ directions respectively, and the Gaussian filter, $G(x, y)$, is given by:

$$
G(x, y)=\frac{1}{2 \pi \sigma^{2}} \exp \left(\frac{x^{2}+y^{2}}{2 \sigma^{2}}\right)
$$

PLBP can be seen as a general case of LBPF and MSLBP differing in that is sampling or no sampling; that the effective areas being tangent or not; and low pass filter types, (more details can be found in [19]).

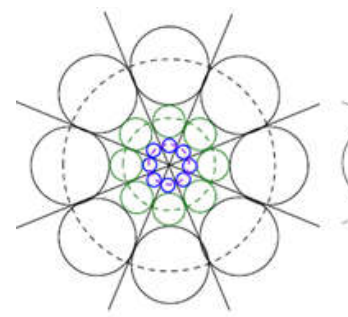

(a)

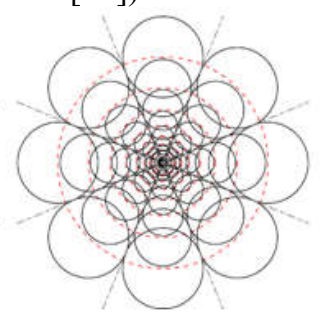

(b)

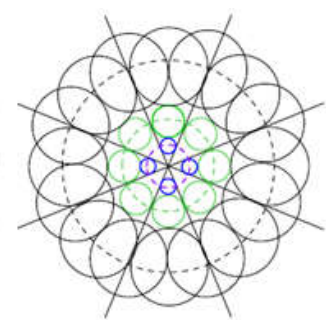

(c)

Fig. 4. Effective areas. (a)LBPF, (b)MSLBP Finer scale space, (c) PLBP with different LBP bits at each scale [19], [10]. 


\section{Bark Datasets}

Two bark image datasets $\left(\mathrm{AFF}^{1}[4]\right.$ and Trunk $\left.12^{2}[16]\right)$ were used to evaluate the multi-scale LBP descriptors. These datasets differ with regard to the number of classes, the number of samples per class, image transformation i.e. scale, pose and illumination changes. Fig. 5 shows samples from each class of the two datasets.
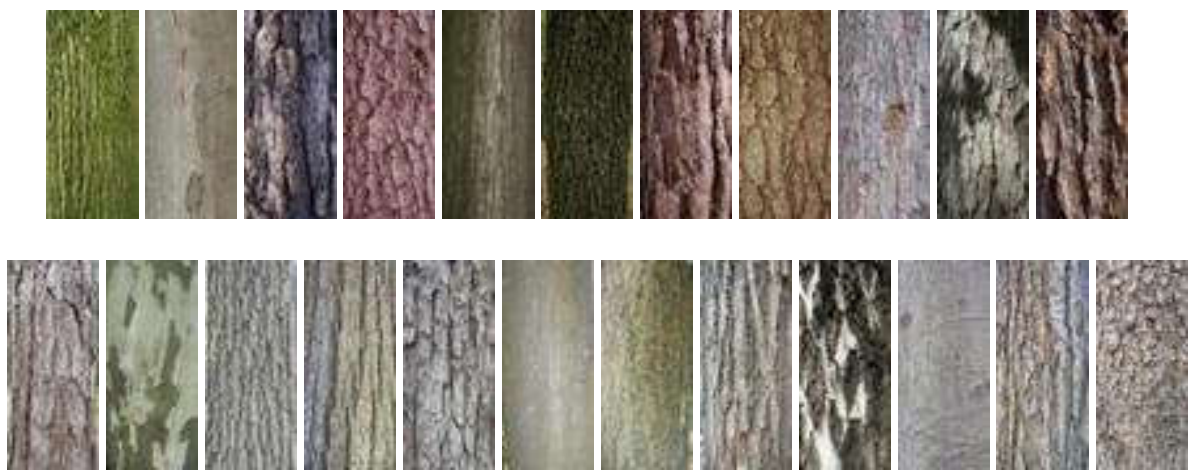

Fig. 5. Bark image samples; Top row, AFF dataset, bottom row, Trunk12 dataset.

- AFF Dataset: The AFF bark dataset provided by "Osterreichische Bundesforste", Austrian Federal Forests (AFF) was first used in [4]. It is a collection of the most common Austrian trees and the dataset contains 1182 bark samples belonging to 11 classes, the size of each class varying between 16 and 213 images. AFF samples are captured at different scales, and under different illumination conditions.

- Trunk12 Dataset: Trunk12 was the first publicly available bark dataset. It contains 393 images of tree barks belonging to 12 different trees that are found in Slovenia. The number of images per class varies between 30 and 45 images. Bark images are captured under controlled scale, illumination and pose conditions. The classes are more homogenous than those of AFF in terms of imaging conditions.

A challenging bark dataset, ImageClef, was excluded from this comparison study since its bark images do not show only the tree trunk, but also contain natural background. Thus a segmentation preprocessing would be required to extract the bark regions. In addition, unlike AFF and Trunk12, ImageClef image samples are subject to rotation changes, as the bark images are captured at random orientations.

1 We are grateful to the computer vision Lab, TU, Vienna for allowing us access to the AFF dataset for scientific use.

2 http://www.vicos.si/Downloads/TRUNK12 


\section{Experimental Results and Evaluation}

Bark images in the AFF dataset and the Trunk12 dataset are of high resolution and processing the samples with their original pixel resolution slows down the task. Therefore, all the images are resized to $600 \times 400 \mathrm{px}$ and converted to gray level.

LBP histograms at level $\mathrm{s}$ are normalized to unit length, and then concatenated. Thus the final multi-scale histogram length is $2^{P} \times s$ where $\mathrm{P}$ is set to 8 neighbors for all scales and $s \in\{1, \ldots, S\}$ where $S=6$, is the scale space level. Table 1 summarizes the parameter settings for each multi-scale LBP descriptor at each level of the scale space, where $\sigma$, and $R_{s}$ denote respectively, the standard deviation of the Gaussian filter and the LBP radius at level s (see $[10,18]$ for details).

Table 1. Multi-scale LBP descriptor parameters over different scales.

\begin{tabular}{lll|ll|l|l}
\hline \multicolumn{3}{l}{ LBPF } & \multicolumn{2}{l|}{ MSLBP } & PLBP & MBLBP \\
\cline { 2 - 7 } $\mathbf{S}$ & $\boldsymbol{\sigma}$ & $\mathbf{R}_{\mathbf{s}}$ & $\boldsymbol{\sigma}$ & $\mathbf{R}_{\mathbf{s}}$ & Image size & Filter size \\
\hline $\mathbf{1}$ & 0.38 & 2.43 & 0.28 & 1.41 & $600 \times 400$ & $3 \times 3$ \\
$\mathbf{2}$ & 0.85 & 5.44 & 0.39 & 2 & $300 \times 200$ & $9 \times 9$ \\
$\mathbf{3}$ & 1.91 & 12.19 & 0.55 & 2.83 & $150 \times 100$ & $15 \times 15$ \\
$\mathbf{4}$ & 4.27 & 27.3 & 0.78 & 4 & $75 \times 50$ & $21 \times 21$ \\
$\mathbf{5}$ & 9.56 & 61.16 & 1.1 & 5.66 & $38 \times 25$ & $27 \times 27$ \\
$\mathbf{6}$ & 21.42 & 136.98 & 1.56 & 8 & $19 \times 13$ & $33 \times 33$ \\
\hline
\end{tabular}

To evaluate the descriptors presented above, we use the Mean Average Precision and the Precision $\backslash$ Recall curves. The Precision (Pre) and the Recall (Rec) are given by:

$$
\text { Pre }=\frac{\# \text { relevant images }}{\text { \#retrieved images }} ; \text { Rec }=\frac{\text { \#retrieved relevant images }}{\text { \#relevant images }}
$$

The Mean Average Precision (MAP) is the mean of the Average Precision (AP) of a set of queries $\mathrm{Q}$, and is defined by:

$$
M A P=\frac{\sum_{q=1}^{Q} A P(q)}{Q}, \text { where } A P(q)=\frac{\sum_{k=1}^{n} \operatorname{Pre}(k) \times f(k)}{\text { \#retrieved relevant images for } q}
$$

Where Pre (k) is the precision at a given cut-off, and $f(k)$ is set to 1 if the retrieved image at rank $\mathrm{k}$ is relevant; zero otherwise.

In what follows, we highlight the max values of MAP in bold and min values in italics.

Table 2. Mean Average Precision of LBP variants without filtering.

\begin{tabular}{llll}
\hline & LBP & LBPu2 & MResLBP \\
\hline AFF & 31.46 & 31.00 & $\mathbf{3 2 . 6 9}$ \\
Trunk12 & $\mathbf{3 1 . 4 2}$ & 29.69 & 30.03 \\
\hline
\end{tabular}


Table 2 reports the MAP values of LBP, LBPu2, and MResLBP. We can see that the LBP and LBPu2 results are very close. The MResLBP, which is the concatenation of three uniform LBPs slightly, exceeds the uniform LBP performance. One reason for this slight improvement of MResLBP, is the aliasing effect in the second and third scales.

Table 3. Mean Average Precision of multi-scale LBP variants on the AFF dataset.

\begin{tabular}{c|lll|lllll|ll}
\hline AFF & \multicolumn{2}{|l}{ MBLBP } & & \multicolumn{2}{l|}{ LBPF } & \multicolumn{2}{l|}{ MSLPB } & \multicolumn{2}{l}{ PLBP } \\
\hline $\mathbf{s}$ & org & $\boldsymbol{u} \mathbf{2}$ & $\boldsymbol{n}$ & $\boldsymbol{o r g}$ & $\boldsymbol{u} \mathbf{2}$ & $\boldsymbol{o r g}$ & $\boldsymbol{u} \mathbf{2}$ & org & $\boldsymbol{u} \mathbf{2}$ \\
\hline $\mathbf{1}$ & 32.12 & 30.28 & 26.39 & 34.1 & 33.95 & 32.14 & 31.66 & 34.99 & 33.69 \\
$\mathbf{2}$ & $\mathbf{3 3 . 8 6}$ & 31.75 & 29.4 & 35.62 & 34.96 & 33.25 & 33.08 & $\mathbf{3 5 . 1 7}$ & 34.18 \\
$\mathbf{3}$ & 33.40 & $\mathbf{3 1 . 9}$ & 29.98 & $\mathbf{3 6 . 6}$ & $\mathbf{3 5 . 3 9}$ & 34.09 & 33.74 & 34.68 & $\mathbf{3 4 . 7 8}$ \\
$\mathbf{4}$ & 32.74 & 31.72 & $\mathbf{3 0 . 0 4}$ & 35.5 & 34.19 & 34.87 & 34.53 & 32.93 & 32.78 \\
$\mathbf{5}$ & 32.17 & 31.65 & 30 & 29.75 & 28.44 & 35.33 & 34.71 & 27.82 & 27.91 \\
$\mathbf{6}$ & 31.67 & 31.53 & 29.93 & 18.97 & 19.91 & $\mathbf{3 5 . 8}$ & $\mathbf{3 4 . 7 7}$ & 22.71 & 22.98 \\
\hline
\end{tabular}

Table 4. Mean Average Precision of multi-scale LBP variants on the Trunk12 dataset.

\begin{tabular}{c|lll|lllll|ll}
\hline Trunk12 & \multicolumn{2}{l}{ MBLBP } & \multicolumn{2}{l|}{ LBPF } & \multicolumn{2}{l}{ MSLPB } & \multicolumn{2}{l}{ PLBP } \\
\hline $\mathbf{s}$ & org & $\boldsymbol{u} \mathbf{2}$ & $\boldsymbol{n}$ & org & $\boldsymbol{u} \mathbf{2}$ & org & $\boldsymbol{u} \mathbf{2}$ & org & $\boldsymbol{u} \boldsymbol{2}$ \\
\hline $\mathbf{1}$ & 32.34 & 28.32 & 24.76 & 32.85 & 31.11 & 31.3 & 28.75 & 36.23 & 34.74 \\
$\mathbf{2}$ & 34.43 & 29.16 & 28.75 & 37.44 & 35.51 & 31.62 & 29.43 & 40.93 & 39.67 \\
$\mathbf{3}$ & 35.8 & 29.84 & 31.22 & 42.8 & 40.14 & 32.68 & 30.59 & 45.05 & 44.29 \\
$\mathbf{4}$ & 36.46 & 30.74 & 32.58 & $\mathbf{4 7 . 6 6}$ & $\mathbf{4 4 . 2 8}$ & 35.09 & 33.15 & $\mathbf{4 7 . 1}$ & $\mathbf{4 6 . 7 4}$ \\
$\mathbf{5}$ & $\mathbf{3 6 . 6 6}$ & 31.22 & 33.32 & 39.07 & 36.64 & 37.38 & 35.01 & 37.6 & 38.27 \\
$\mathbf{6}$ & 36.57 & $\mathbf{3 1 . 7 9}$ & $\mathbf{3 3 . 7 7}$ & 17.38 & 18.2 & $\mathbf{3 9 . 5 9}$ & $\mathbf{3 6 . 6 6}$ & 23.45 & 24.85 \\
\hline
\end{tabular}

Table 3 and Table 4 show MAP values for the multi-scale LBP descriptors on the AFF and the Trunk12 datasets respectively with increasing scale space levels. The uniform extension of multi-scale LBP variants, noted $\boldsymbol{u} 2$, as well as the original patterns i.e. all possible patterns (256), noted org, are also considered. Note that for MBLBP, the uniformity measure defined in [17] is also tested in this study, noted by MBLBPn. Fig 6 illustrates Recall $\backslash$ Precision curves. The multi-scale LBP descriptors are chosen at their best scale according to the highest MAP value in Table 3 and Table 4 for the AFF and Trunk 12 datasets respectively. 
The above results suggest that all multi-scale descriptors at the proper scale outperform MResLBP and basic LBP. That is because in multi-scale LBP descriptors, macro-texture information captured at the high level, contributes to increasing MAP value.

Moreover, the information is collected from regions (effective areas) and not from single pixels, as is performed in MResLBP. Thus reduces noise sensitivity and increases performances.
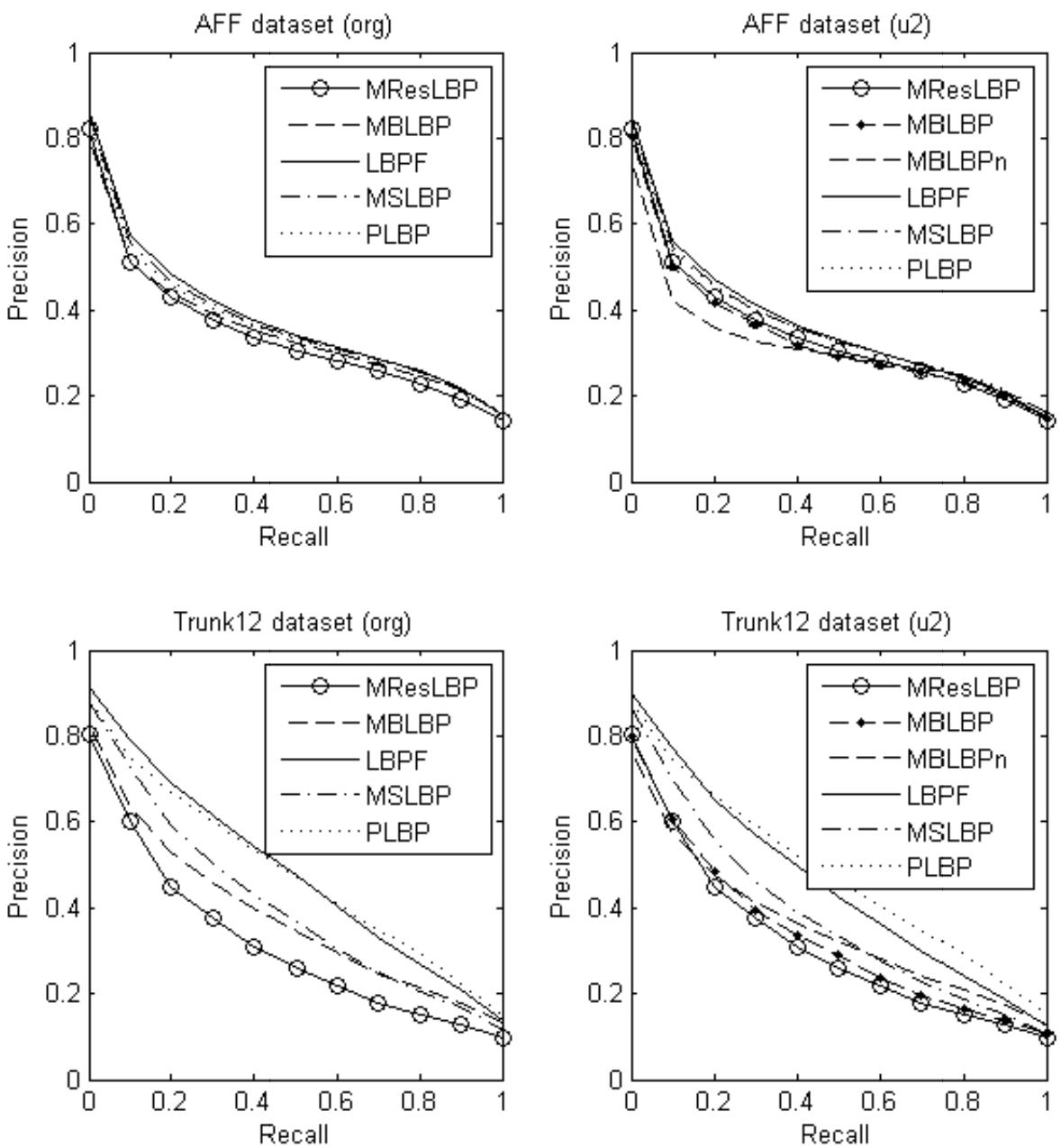

Fig. 6. Recall $\backslash$ Precision curves on the AFF and Trunk12 datasets.

It is clear that more LBP scales lead to increased MAP values on both datasets. However, multi-scale descriptors do not behave in a similar way at different scales. It is worth noting that all the multi-scale LBP descriptors on the Trunk12 dataset behave as well as, or better than they do for the AFF dataset. One reason for this may be that 
Trunk12 classes are more homogenous than those of AFF regarding both imaging conditions and botanical factors.

One reason why all multi-scale descriptors with only $u 2$ patterns perform slightly lower than org patterns is the part of information that is roughly described by the last bin of the non-uniform histogram.

MBLBPn has the lowest MAP value and the worst Recall $\backslash$ Precision curve on the AFF dataset, but not on the Trunk12 dataset. Note, however, that MBLBPn is based on a statistical distribution of patterns and not on fundamental local texture properties. Consequently, the comparison of two histograms can match completely different patterns together. This can explain the AFF results. For the Trunk12 dataset, we suppose that the matching between different patterns coincides with space projection.

MBLBP has the lowest performances on the AFF dataset and the Trunk12 dataset. We believe that this came from low pass filtering used, i.e. mean filter in MBLBP.

For MSLBP, the best MAP values are obtained at scale 6 on both datasets. MSLBP MAP values increase slowly compared to other variants. Indeed, a finer scale with step of $\sqrt{2}$ is used between LBP radii (see Table 1) and Gaussian smoothing is applied firstly at scale 4 (more details in [10]). The best results for LBPF are obtained at scale 3 with LBP radius equal to 12.19, which is higher than the MSLBP radius at scale 6 $\left(\mathrm{R}_{6}=8\right)$. Based on these remarks, we believe that MSLBP reaches its optimal performance at scales higher than 6. However, it results in a longer histogram compared to LBPF.

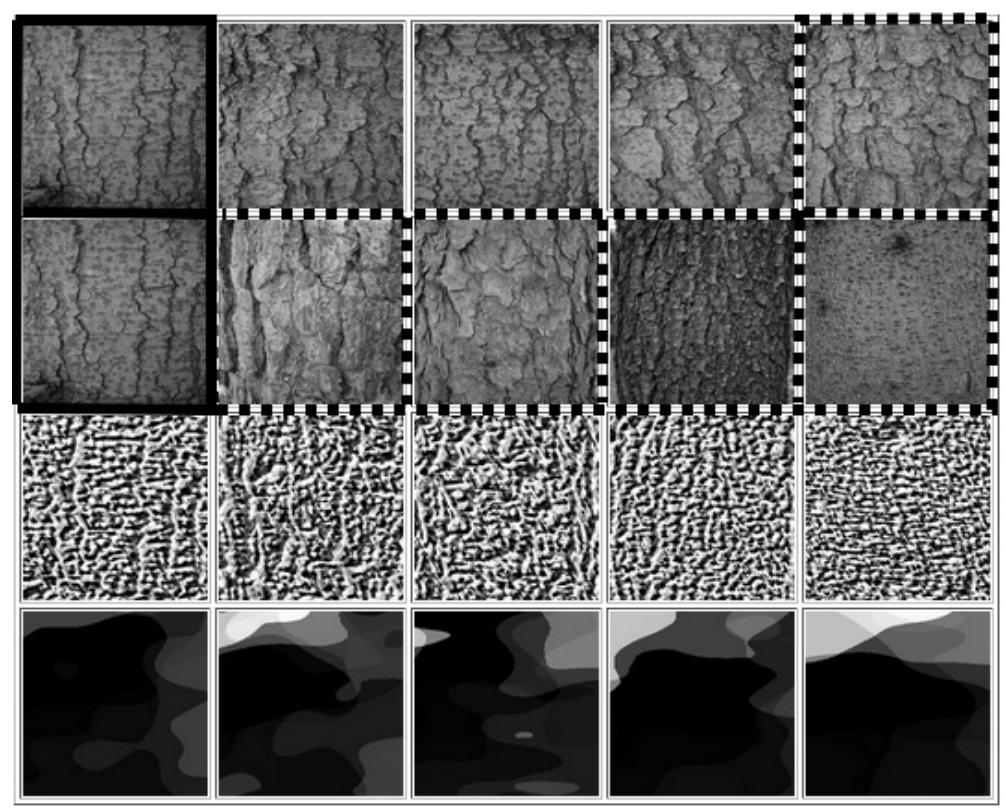

Fig. 7. LBPF descriptor behavior. Request image highlighted in solid square, false positive in dotted square, from top to bottom; first five returned images at level 3, second row, returned images et level 6; third row: LBP images at level 3, with high inter-class variation; bottom row, LBP images at level 6 , high inter-class similarity. 
The sharp drop in MAP values for the LBPF descriptor on both, AFF and Trunk 12 from scales 3 and 4 respectively can be explained as follows: With increasing values of $\sigma$ over the scales (details in Table 1), images are increasingly smooth, and local texture information is lost and subject to noise sensitivity. Thus the descriptor loses its discriminative power. Fig 7 shows an example of an image request and the first 5 images returned, the LBPF descriptor, cannot distinguish between different classes at scale level 6.

The deterioration in PLBP performances on the two datasets is due to the fact that images at coarse pyramid levels are of very low resolution, as reported in Table 1, and do not provide enough structure information.

\section{Conclusion}

This paper reports on the evaluation results of basic LBP, uniform LBP, and five multi-scale LBP descriptors. These descriptors are tested and compared on two bark datasets (AFF and Trunk12).

It is worth noticing that multi-scale LBP provides more discriminative texture features than basic and uniform LBP. Therefore, multi-scale information improves LBP results. LBPF gives the best results over all the descriptors tested on both datasets. MBLBPn produced less satisfying results than we had expected for both datasets. This is due to its uniformity definition. Using a distribution percentage does not take into account the local structure properties, and the uniqueness of the relative patterns.

In this work, we focused on the multi-scale problem. It would be interesting to explore other LBP configurations with different neighborhood topologies in addition to the classic circular one, and to combine other complementary texture information in order to boost performances.

\section{References}

1. Belhumeur, P.N., Chen, D., Feiner, S., Jacobs, D.W., Kress, W.J., Ling, H., Lopez, I., Ramamoorthi, R., Sheorey, S., White, S., Zhang, L.: Searching the World's Herbaria: A System for Visual Identification of Plant Species. In: Forsyth, D., Torr, P., and Zisserman, A. (eds.) Computer Vision - ECCV 2008. pp. 116-129. Springer Berlin Heidelberg (2008).

2. Nilsback, M.E.: An automatic visual flora: Segmentation and classification of flower images, http://www.robots.ox.ac.uk/ vgg/publications/2009/Nilsback09/, (2009).

3. Chi, Z., Houqiang, L., Chao, W.: Plant species recognition based on bark patterns using novel Gabor filter banks. Proceedings of the 2003 International Conference on Neural Networks and Signal Processing, 2003. pp. 1035-1038 Vol.2 (2003).

4. Fiel, S., Sablatnig, R.: Automated identification of tree species from images of the bark, leaves and needles. Proceedings of the 16th Computer Vision Winter Workshop. pp. 67-74. Andreas Wendel, Sabine Sternig, Martin Godec, Mitterberg, Austria (2011).

5. Goëau, H., Bonnet, P., Barbe, J., Bakic, V., Joly, A., Molino, J.-F., Barthelemy, D., Boujemaa, N.: Multi-organ Plant Identification. Proceedings of the 1st ACM International Workshop on Multimedia Analysis for Ecological Data. pp. 41-44. ACM, New York, NY, USA (2012). 
6. Song, J., Chi, Z., Liu, J., Fu, H.: Bark classification by combining grayscale and binary texture features. Proceedings of 2004 International Symposium on Intelligent Multimedia, Video and Speech Processing, 2004. pp. 450-453 (2004).

7. Wan, Y.-Y., Du, J., Huang, D.-S., Chi, Z., Cheung, Y., Wang, X.-F., Zhang, G.-J.: Bark texture feature extraction based on statistical texture analysis. Proceedings of 2004 International Symposium on Intelligent Multimedia, Video and Speech Processing, 2004. pp. 482485 (2004).

8. Huang, Z.-K., Quan, Z.-H., Du, J.-X.: Bark Classification Based on Contourlet Filter Features Using RBPNN. In: Huang, D.-S., Li, K., and Irwin, G.W. (eds.) Intelligent Computing. pp. 1121-1126. Springer Berlin Heidelberg (2006).

9. Sixta, T.: Image and Video-based Recognition of Natural Objects, Diploma Thesis, Prague: Czech Technical University (2011).

10. Sulc, M., Matas, J.: Kernel-mapped histograms of multi-scale LBPs for tree bark recognition. 2013 28th International Conference of Image and Vision Computing New Zealand (IVCNZ). pp. 82-87 (2013).

11. Whitmore, T.C.: Studies in Systematic Bark Morphology. I. Bark Morphology in Dipterocarpaceae. New Phytol. 61, 191-207 (1962).

12. Mirmehdi, M., Xie, X., Suri, J.: Handbook of Texture Analysis. Imperial College Press, London, UK, UK (2009).

13. Huang, Z.-K., Huang, D.-S., Lyu, M.R., Lok, T.-M.: Classification Based on Gabor Filter Using RBPNN Classification. 2006 International Conference on Computational Intelligence and Security. pp. 759-762 (2006).

14. Huang, Z.-K.: Bark Classification Using RBPNN Based on Both Color and Texture Feature, (2006)

15. Goëau, H., Joly, A., Bonnet, P., Selmi, S., Molino, J.-F., Barthélémy, D., Boujemaa, N.: LifeCLEF Plant Identification Task 2014. In: Cappellato, L., Ferro, N., Halvey, M., and Kraaij, W. (eds.) CLEF2014 Working Notes. Working Notes for CLEF 2014 Conference, Sheffield, UK, September 15-18, 2014. pp. 598-615. CEUR-WS (2014).

16. Švab, M.: Computer-vision-based tree trunk recognition, Bsc Thesis, (Mentor: doc. dr. Matej Kristan), Fakulteta za racunalništvo in informatiko, Univerza v Ljubljani, (2014)

17. Liao, S., Zhu, X., Lei, Z., Zhang, L., Li, S.Z.: Learning Multi-scale Block Local Binary Patterns for Face Recognition. In: Lee, S.-W. and Li, S.Z. (eds.) Advances in Biometrics. pp. 828-837. Springer Berlin Heidelberg (2007).

18. Mäenpää, T., Pietikäinen, M.: Multi-scale Binary Patterns for Texture Analysis. Proceedings of the 13th Scandinavian Conference on Image Analysis. pp. 885-892. SpringerVerlag, Berlin, Heidelberg (2003).

19. Qian, X., Hua, X.-S., Chen, P., Ke, L.: PLBP: An effective local binary patterns texture descriptor with pyramid representation. Pattern Recognit. 44, 2502-2515 (2011).

20. Ojala, T., Pietikäinen, M., Mäenpää, T.: Multiresolution Gray-Scale and Rotation Invariant Texture Classification with Local Binary Patterns. IEEE Trans Pattern Anal Mach Intell. 24, 971-987 (2002).

21. Topi, M., Timo, O., Matti, P., Maricor, S.: Robust texture classification by subsets of local binary patterns. 15th International Conference on Pattern Recognition, 2000. Proceedings. pp. 935-938 vol.3 (2000). 